La combinaison des égalités (43), (44), (58), donne encore la relation

$$
\begin{gathered}
\frac{1}{\rho_{\zeta_{0}}^{1}}=\frac{\left(l-l_{0}\right) \cos \lambda_{0} \sin \zeta_{0}-\left(\lambda-\lambda_{0}\right) \cos \zeta_{0}}{s} \\
+\frac{s}{2} \operatorname{tang} \lambda_{0} \sin ^{2} \zeta_{0} \cos \zeta_{0}-\frac{s^{2}}{6} \sin ^{2} \zeta_{0}\left(\frac{\cos ^{2} \zeta_{0}}{\cos ^{2} \lambda_{0}}-2 \sin ^{2} \zeta_{0}\right) .
\end{gathered}
$$

(Continua).

\title{
EXTRAIT D'UNE LETTRE
}

DE M. K R ONE CKERA M. BRIOSCHI.

.... Sans doute l'un et l'autre des systèmes de substitutions (A, $\left.\mathbf{A}^{\prime}\right)$ dont Mr. Hermite fail mention ${ }^{*}$ ) est propre à définir le type d'une fonclion de sept lettres possédant trente valeur's distinctes. Mais c'est un seul type auquel les deux systèmes conviennent, savoir celui que j'ai publié dans mon mémoire du 22. Avril 1858***). En effet, la fonction de $a, b, c, d, e, f, g$ que j'y ai donnée reste invariable par les substitutions du système $\mathbf{A}$ ou par celles du système $\mathbf{A}$ ', suivant que les lettres $a, b$, $c, d, e, f, g$ sont remplacées respectivement par : $x_{0}, x_{6}, x_{5}, x_{4}, x_{3}, x_{2}, x_{x}$ ou par: $x_{0}, x_{1}, x_{2}, x_{3}, x_{4}, x_{5}, x_{6}$. Donc les deux systemes $\mathbf{A}$ et $\mathbf{A}^{\prime}$ ne sont pas essentiellement distincts, bien qu'ils se présentent sous des formes différentes.

Pour donner quelques éclaircissements sur ce point; considérons une substitulion quelconque de $n$ lettres : $a, b, c, \ldots k$. En faisant :

$$
a=x_{1}, b=x_{2}, c=x_{3}, \ldots k=x_{n}
$$

la substitution dont il s'agit pourra s'exprimer par un changement: $\left(\begin{array}{c}i \\ \theta(i)\end{array}\right)$ opéré sur les indices. Or la méme substitution sera représentée par: $\left(\begin{array}{l}\varphi(i) \\ \varphi \theta(i)\end{array}\right)$, si l'on pose:

$$
a=x_{p(1)}, b=x_{\%(2)}, c=x_{p(3)}, \ldots k=x_{\xi(n)}
$$

$x_{\varphi(1)}, x_{\varphi(2)}, \ldots x_{\phi(n)}$ élant une quelconque des $1.2 .3 \ldots n$ permutations, dont les quanttités: $x_{1}, x_{2}, \ldots x_{n}$ sont susceptibles. Enfin il est clair que, $\psi(i)$ désignant la fonction inverse de $\varphi(i)$, le changement $\left(\begin{array}{c}\varphi(i) \\ \varphi \theta(i)\end{array}\right)$ est identique avec celıi-ci : $\left(\begin{array}{c}i \\ \varphi \theta \psi(i)\end{array}\right)$.

La mème substitution des $n$ leltres : $a, b, c, \ldots k$ peut done ètre représenté. sous 1. 2.3...n formes différentes:

$$
\left(\begin{array}{c}
i \\
\theta(i)
\end{array}\right),\left(\begin{array}{c}
i \\
\varphi \theta \psi(i)
\end{array}\right), \quad\left(\begin{array}{c}
i \\
\varphi_{\mathbf{1}} \theta \psi_{\mathrm{I}}(i)
\end{array}\right), \quad\left(\begin{array}{c}
i \\
\psi_{2} \theta \psi_{2}(i)
\end{array}\right), \ldots
$$

C'est par ce rapprochement qu'on parvient à réuuir les deux systèmes de substitutions $\left(\mathbf{A} ; \mathbf{A}^{\prime}\right)$ de Mr. Hermite. En effet, si l'on désigne une substitution quelconque du système A par: $\left(\begin{array}{c}i \\ \theta(i)\end{array}\right)$, celles du système $\mathbf{A}^{\prime}$ sont exprimées par: $\left(\begin{array}{c}i \\ -\theta(-i)\end{array}\right)$. ce qui est le cas particulier de $:\left(\begin{array}{c}i \\ \varphi \theta \psi(i)\end{array}\right)$, où l'ón a fait : $\varphi(i)=-i$. 